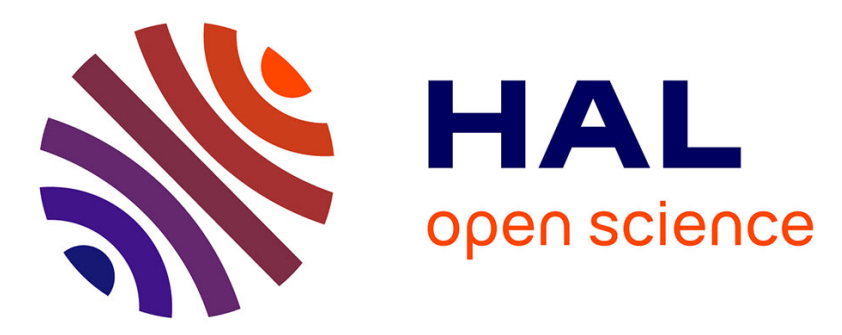

\title{
Cluster Agglomeration Induced by Dust-Density Waves in Complex Plasmas
}

Simon Dap, David Lacroix, Robert Hugon, Ludovic de Poucques, Jean-Luc

Briancon, Jamal Bougdira

\section{- To cite this version:}

Simon Dap, David Lacroix, Robert Hugon, Ludovic de Poucques, Jean-Luc Briancon, et al.. Cluster Agglomeration Induced by Dust-Density Waves in Complex Plasmas. Physical Review Letters, 2012, 109 (24), 10.1103/PhysRevLett.109.245002 . hal-02314410

\author{
HAL Id: hal-02314410 \\ https://hal.science/hal-02314410
}

Submitted on 12 Oct 2019

HAL is a multi-disciplinary open access archive for the deposit and dissemination of scientific research documents, whether they are published or not. The documents may come from teaching and research institutions in France or abroad, or from public or private research centers.
L'archive ouverte pluridisciplinaire HAL, est destinée au dépôt et à la diffusion de documents scientifiques de niveau recherche, publiés ou non, émanant des établissements d'enseignement et de recherche français ou étrangers, des laboratoires publics ou privés. 


\title{
Cluster Agglomeration Induced by Dust-Density Waves in Complex Plasmas
}

\author{
Simon Dap, ${ }^{1, *}$ David Lacroix,${ }^{2, \dagger}$ Robert Hugon, ${ }^{1}$ Ludovic de Poucques, ${ }^{1}$ Jean-Luc Briancon, ${ }^{1}$ and Jamal Bougdira ${ }^{1}$ \\ ${ }^{1}$ Institut Jean Lamour, Département CP2S UMR 7198 CNRS, Université de Lorraine, \\ BP 70239, 54506 Vandoeuvre-les-Nancy Cedex, France \\ ${ }^{2}$ LEMTA, UMR 7563 CNRS, Faculté des Sciences et Technologies, Université de Lorraine, \\ BP 70239, 54506 Vandoeuvre-les-Nancy Cedex, France \\ (Received 6 September 2012; published 10 December 2012)
}

\begin{abstract}
Experimental results showing the agglomeration of large carbonaceous particles in a dusty plasma are reported. Experiments were performed in a capacitively coupled $\mathrm{rf}$ argon plasma. Acetylene was injected to produce dust particles. When a sufficient amount of nanoparticles is present in the cathodic sheath, selfexcited dust-density waves occur. The latter ones induce the motion of larger clusters, which vertically oscillate with the displacement of wave fronts. In some cases, the relative velocity of large particles was high enough to overcome the Coulomb repulsion forces, and agglomeration can be observed. The mechanisms underlying this process are discussed.
\end{abstract}

DOI: 10.1103/PhysRevLett.109.245002

PACS numbers: 52.27.Lw, 52.35.Fp, 52.70.Kz

Several theoretical and experimental studies dealing with particle growth in dusty plasmas were carried out over the last few decades. These particular media can be encountered in several environments, such as planetary atmospheres, plasma processing, and fusion plasma.

In low pressure reactive plasmas, e.g., argon-acetylene plasmas, the dust growth mechanism is well described as long as particles sizes remain small [1]. In this case, acetylene dissociation produces a large amount of molecular precursors. Then, a nucleation phase occurs and nanometric particles are accumulated to a high density. Due to their great number, nanoscale particles are either weakly or not at all charged [2]. The electrostatic repulsion between single particles is thus small. It allows the beginning of a second rapid growth phase characterized by agglomeration between nanoparticles. As particles grow, their density decreases and they carry an increasing number of negative charges. When a critical size is reached, Coulomb repulsions prevent further agglomeration events and dust particles slowly grow due to the sticking of molecular species to their surface. This is the third growth phase. However, several experiments $[3,4]$ have reported that clusters of particles larger than this critical size can be formed in dusty plasmas. Many theoretical models were proposed in order to explain this phenomenon. For some of them, a "shadowing force" [5-7] due to ion collisions with dust might be large enough to overcome the electrostatic repulsion. This mechanism is likely to arise at high ion density and low pressure. Another theory lies on the "polarizationinduced ion flux asymmetry" model [8,9]. In the latter case, when two particles are close enough, the electric field of a dust induces the polarization of the neighbouring dust. As a consequence, the ion fluxes towards the particle surface are asymmetric and this might lead them to agglomerate. Lastly, in a recent study by Du et al. [10], the assumption of agglomeration induced by "dust-density waves" (DDWs) was reported. The authors proposed that DDWs can sufficiently accelerate the negatively charged dust particles to promote their agglomeration. If particle velocities are high enough, Coulomb repulsion can be overcome and short distance interactions (i.e., van der Walls, polarization effects, and spatial discreteness of the particle charge) could arise. Authors have checked the reliability of this assumption, but, due to its low probability, no agglomeration event (collision followed by sticking) has been directly observed during their experiments. In the present work, a dedicated experiment was built in order to observe such an event. For the first time, the agglomeration, triggered by DDWs, of large carbonaceous dust was recorded. These observations support the model proposed in Ref. [10] about large particles bonding in a low pressure plasma where DDWs occur.

In this Letter, we address carbonaceous dust cluster agglomeration in the cathodic sheath of a rf argon- $\mathrm{C}_{2} \mathrm{H}_{2}$ plasma. To achieve this goal, a dedicated reactor with a parallel plate asymmetrically driven $\mathrm{rf}$ discharge at 13.56 MHz was used (Fig. 1). The upper electrode (cathode) is biased through to an impedance matching network, whereas the lower electrode (anode) is grounded. We use argon gas at pressures between 50 and 500 mTorr. The delivered rf power is usually in the range of 10 to $50 \mathrm{~W}$. In order to produce dust, acetylene gas is injected inside the reaction chamber. Gases are continuously injected and pumped from the top and bottom of the reactor, respectively. Gas flow rates are usually in the ratio $Q_{\mathrm{Ar}} / Q_{\mathrm{C}_{2} \mathrm{H}_{2}}=$ $12 \mathrm{sccm} / 2 \mathrm{sccm}$. Unlike in Ref. [10], there is no injection of calibrated particles in the discharge during the reported experiments. Concerning the imaging devices, the interelectrode region is illuminated by a vertical diode laser sheet at $\lambda=639 \mathrm{~nm}$. The electrode axis is in a cut plane of the laser sheet. The light scattered by dust particles is recorded with a Photron Fastcam-SA1 that is set perpendicularly to 


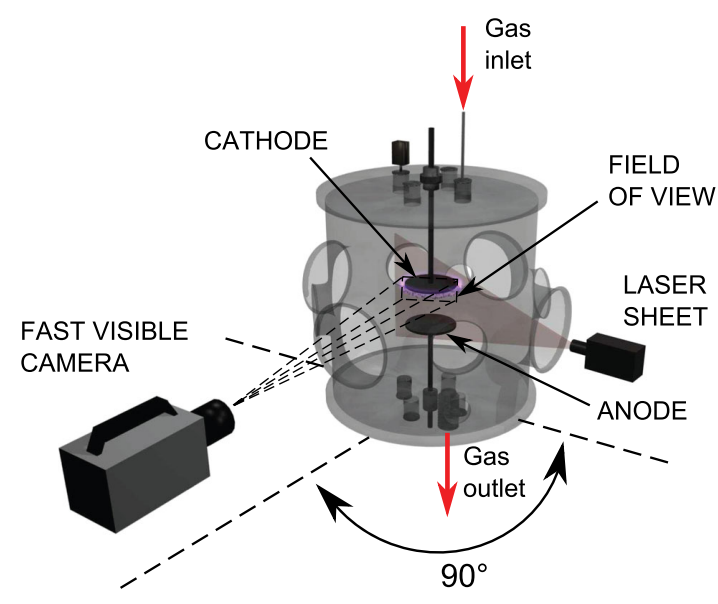

FIG. 1 (color online). Overview of the experimental setup. The chamber characteristic dimensions are height $h_{c}=36 \mathrm{~cm}$ and diameter $D_{c}=36.5 \mathrm{~cm}$. The electrode diameters are $D_{e}=5 \mathrm{~cm}$; the space between the cathode and the anode is $h_{e}=6.5 \mathrm{~cm}$. There are eight $\mathrm{KB} 7$ windows (4 large, $D_{\mathrm{wl}}=$ $10 \mathrm{~cm}$, and 4 small, $D_{\mathrm{ws}}=5 \mathrm{~cm}$ ) evenly spaced on the side of the reactor.

the laser light sheet. The acquisition frame rate is $5000 \mathrm{fps}$ for a spatial resolution of $768 \times 496$ pixels. A lens (Nikon AF Micro-Nikkor $200 \mathrm{~mm} f / 4 \mathrm{D}$ IF-ED) is focused on a small area of the cathodic sheath. The former is a macrolens allowing a spatial resolution of $30 \mu \mathrm{m} / \mathrm{pixel}$. A dedicated image processing tool (TRACE) was developed at the laboratory to track the motion of dust particles.

In this work, dust is produced by acetylene molecule dissociation instead of injecting calibrated dust particles, as done in Ref. [10]. It allows the fast generation of nanometric particles with diameters around 100 nanometers in a few seconds. The first steps of the agglomeration process presumably obey the classical model proposed by Watanabe [1], which was previously mentioned. The particles are trapped inside the cathodic sheath through the balance between downward forces, due to electric and gravity fields, and upward force, due to the ion drag. When a sufficient density of nanometric dust particles is reached in the cathodic sheath, self-excited DDWs spontaneously occur within the dust cloud (Fig. 2).

DDWs are probably excited by an ion-dust streaming instability [11-13]. Waves propagate in the direction of the electric field from the plasma to the cathode. Wave fronts, corresponding to regions of high nanometric particle density, appear as bright bands on Fig. 2. Eventually, agglomerates (large spots in Fig. 2) made of a high number of nanoparticles can be observed at the top of the cathodic sheath, close to the electrode. They are carried by the wave fronts, as suggested in Ref. [4].

Each movie (see Fig. 2) recorded by the fast camera can be analysed with a periodgram [13], as depicted in Fig 3. The latter is constructed by the juxtaposition of a vertical pixel line (cathode axis) extracted from each frame of the

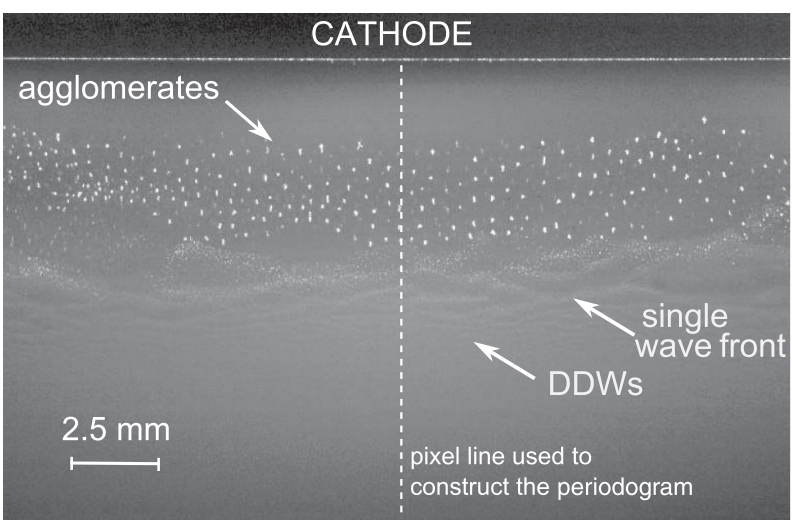

FIG. 2. Particles cloud in the cathodic plasma sheath. Agglomerates (large spots) and DDWs at $t=43 \mathrm{~s}$ after acetylene injection. Operating conditions: $p=100 \mathrm{mTorr}, P_{\mathrm{rf}}=10 \mathrm{~W}$, $Q_{\mathrm{Ar}} / Q_{\mathrm{C}_{2} \mathrm{H}_{2}}=12 \mathrm{sccm} / 2 \mathrm{sccm}$.

registered movie. Thus, the spatial and temporal behavior of DDWs can be addressed. On this figure, three distinct areas can be identified. The lower one (called region 1), where only nanometric particles are present; the intermediate region (called region 2), where large agglomerates are located; and the upper dust-free area close to the cathode (called region 3). Mean dispersion parameters of DDWs such as wavelength, frequency, and phase velocity can be appraised with region 1 of the periodgram through the study of the successive wave fronts. In this case, we found $\lambda_{\mathrm{DDW}}=0.7 \mathrm{~mm}, f_{\mathrm{DDW}}=232.6 \mathrm{~Hz}$, and

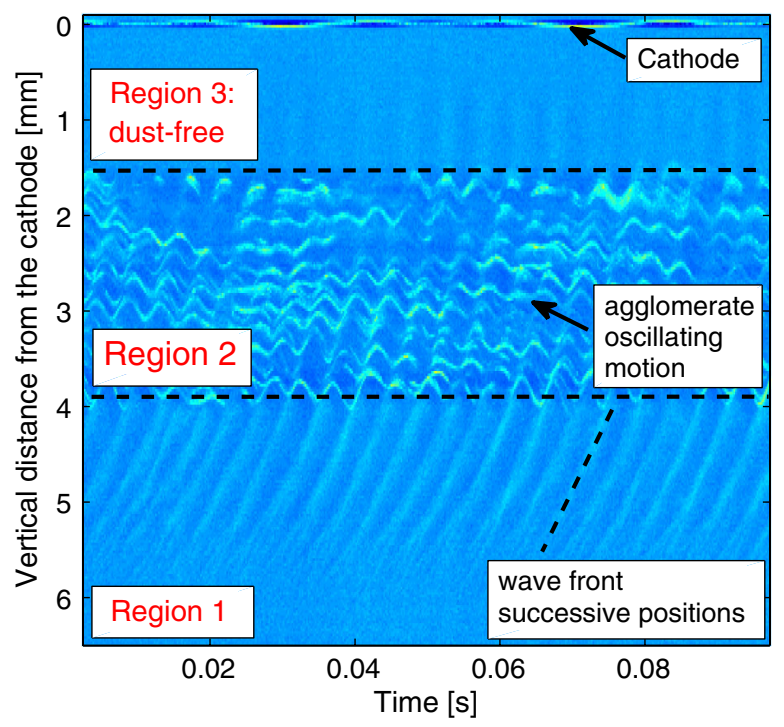

FIG. 3 (color online). Periodgram of waves and agglomerates below the cathode along its vertical axis at $t=150 \mathrm{~s}$ after acetylene injection. $x$ axis, time; $y$ axis, distance from the cathode. Region 1, DDW wave fronts; region 2, oscillating agglomerates. Operating conditions: $p=180 \mathrm{mTorr}, P_{\mathrm{rf}}=$ $25 \mathrm{~W}, Q_{\mathrm{Ar}} / Q_{\mathrm{C}_{2} \mathrm{H}_{2}}=12 \mathrm{sccm} / 2 \mathrm{sccm}$. 
$v_{\text {DDW }}=15.8 \mathrm{~cm} / \mathrm{s}$. In region 2 , sinusoidal trajectories of agglomerates can be seen. They correspond to the vertical oscillations of large agglomerates in the upper part of the cathodic sheath. The smallest nanoparticles cannot be detected in region 2 with the fast camera. However, light extinction measurements confirm their presence [14]. At the interface between the two regions, it can be noticed that DDW wave fronts carry the large agglomerates toward the cathode. As they are moving closer to the cathode, the electric field forces them down until they reach a new wave front. In order to better understand the agglomeration process of large dust in region 2 , another periodgram focused on this area with a larger oscillation motion of dust is proposed in Fig. 4 (with the same operating conditions as in Fig. 2). Much information can be extracted from this plot. First, the velocities of the large dust particles during their rising and falling motions are different, the falling velocity being larger than the rising one. Then, as large dust particles are falling towards the incoming wave front just below, their motion slows down due to electrostatic repelling. When they have reached this lower wave front, they partly eject the present agglomerates, which were going up with the DDW wave front, and make them go downwards. Then, they are carried by this new wave front and restart this oscillating cycle. The movie corresponding to the motion of DDWs and aggregates is given in the Supplemental Material [15].

In addition, it can be noted that sometimes the interaction between two large dust particles leads to agglomeration. In the enlargement of Fig. 4, two oscillating particles

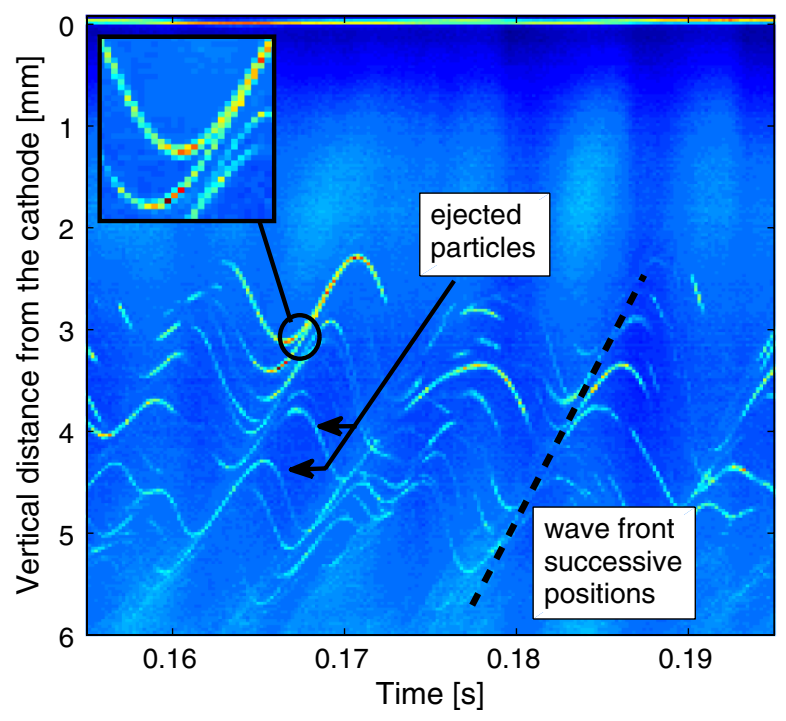

FIG. 4 (color online). Periodgram of agglomerates below the cathode along its vertical axis at $t=26 \mathrm{~s}$ after acetylene injection. $x$ axis, time; $y$ axis, distance from the cathode. Enlargement: collision between vertically oscillating agglomerates. Operating conditions: $p=100$ mTorr, $P_{\mathrm{rf}}=10 \mathrm{~W}$, $Q_{\mathrm{Ar}} / Q_{\mathrm{C}_{2} \mathrm{H}_{2}}=12 \mathrm{sccm} / 2 \mathrm{sccm}$. are probably combined into a larger one. In this particular case, both particles were going upwards, but the one underneath was moving faster than the other one. However, this observation requires further examination. Indeed, the spatial resolution of both incoming dust and of the resulting agglomerate is not entirely adequate within the periodgram plot. Thus, to support our conclusions, the tracking of such an event, considering the whole frame at each successive time step, has been done in the cathodic sheath region. In Fig. 5, the motion of two dust particles oscillating under the effect of DDWs and their agglomeration into a larger particle is presented. The movie corresponding to this agglomeration is given in the Supplemental Material [15]. Here, dust tracking has been done with the TRACE algorithm [16], which automatically detects the successive positions of each particle and its respective size. We obtained the size of particle $1, S_{1}=2 \mathrm{px} \times 8 \mathrm{px}$, and the size of particle $2, S_{2}=2-3 \mathrm{px} \times 8 \mathrm{px}$ (one pixel length $l_{\mathrm{px}} \simeq 29.07 \mu \mathrm{m}$ ). The resulting agglomerate of characteristic size $S_{\text {agglo }}=3-4 \mathrm{px} \times 16 \mathrm{px}$ is a perfect superposition of the two previous ones. Let us recall that the motion of each considered particle is within the laser light sheet. This makes such an agglomeration event quite rare because clusters are frequently driven out of this plane. In the present case, the three particles move according to DDW propagation, and their vertical positions vary with time. Regarding the relative velocity between particles 1 and 2 , we measured its decrease from $37 \mathrm{~cm} / \mathrm{s}$ to $23 \mathrm{~cm} / \mathrm{s}$ just before the clustering. Kinetic $E_{c}$ and Coulomb $E_{p}$ energies were appraised on the basis of the relative speed (particle tracking with TRACE) and the assessed charge (orbital motion limited theory) of the involved particles.

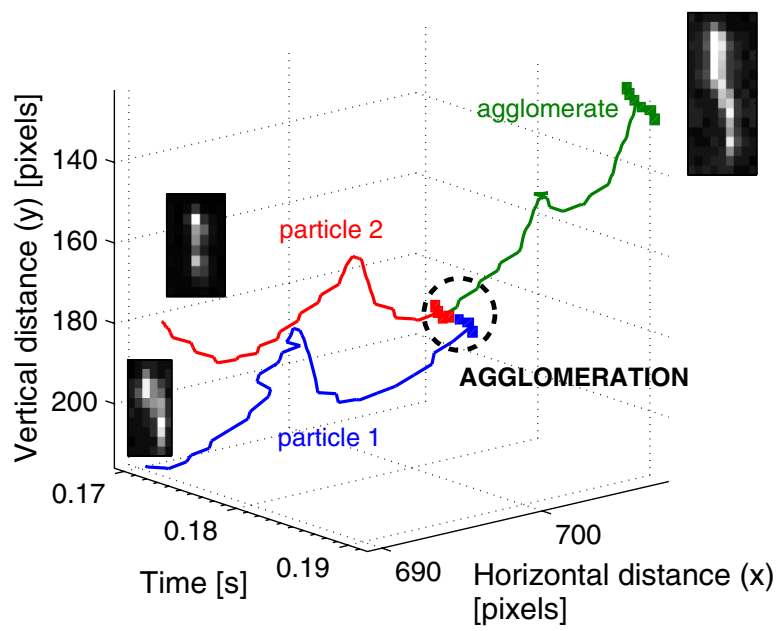

FIG. 5 (color online). Dust motion, collision, and agglomeration below the cathode at $t=35 \mathrm{~s}$ after acetylene injection. $x$ axis, horizontal position; $y$ axis, time; $z$ axis, vertical position. Enlargement: incoming and outgoing particles. Operating conditions: $\quad p=100$ mTorr, $\quad P_{\mathrm{rf}}=25 \mathrm{~W}, \quad Q_{\mathrm{Ar}} / Q_{\mathrm{C}_{2} \mathrm{H}_{2}}=$ $12 \mathrm{sccm} / 2 \mathrm{sccm}$. 
It shows that, just before collision (one time step given by the temporal resolution), $E_{c}$ is at least one order of magnitude larger than $E_{p}$. Lastly, one can notice that agglomeration is done at the extremity of the incoming particles. This point is important and was expected, as it must be the most likely location for agglomeration of large, highly charged particles [17,18]. With this particle orientation, only a fraction of the total particle charges, located at particle extremity, are interacting. Most of the charges carried by the dust particles are shielded by the plasma species. Furthermore, several phenomena can help particles to agglomerate, such as the mechanism suggested by Goertz [19]. When two particles get closer, the overlapping of their Debye shielding clouds leads to a reduction in their charge. As a consequence, the resulting Coulomb repulsion is getting weaker. The frequency of such collision events is quite difficult to appraise. Nevertheless, at least two undisputable agglomeration events have been observed in $0.21 \mathrm{~s}$. Assuming a constant agglomeration rate, the resulting number of agglomeration events could represent $30 \%$ of the total number of agglomerates within the laser sheet (with the total number around 1000 at a movie time of $t=$ $35 \mathrm{~s})$. Observation at longer durations after the acetylene injection even shows that agglomerates can be structured as a long carbonaceous chain vertically oscillating in the cathodic sheath. SEM measurements achieved on agglomerates collected during experiments confirm the occurrence of a one-dimensional growth, vertical and along the electric field in the plasma sheath.

In conclusion, we have experimentally investigated the behavior of large carbonaceous particles set in motion by self-excited DDWs. The dispersion parameters of such DDWs and the motion of dust were identified for a broad range of operating conditions. With a dedicated tracking tool of cluster displacements within the plasma cathodic sheath, we have demonstrated that agglomeration of a large dust particle is possible, as suggested by the work of Du et al. [10]. In the reported case, DDWs sufficiently accelerate incoming particles to overcome the Coulomb repulsion. The resulting agglomerate preserves the shape of the initial dust particles, which were bounded at their extremities. We believe that these results can be of great interest for the community involved in the dusty plasma research.

This study is partially funded by the EURATOM-CEA Association (Contract No. LRC-DSM\#99.18).

*simon.dap@ijl.nancy-universite.fr

†david.lacroix@univ-lorraine.fr

[1] Y. Watanabe, J. Phys. D 39, R329 (2006).

[2] J. Goree, Plasma Sources Sci. Technol. 3, 400 (1994).

[3] J. H. Chu, Ji-Bin Du, and Lin I, J. Phys. D 27, 296 (1994).

[4] M. Schwabe, M. Rubin-Zuzic, S. Zhdanov, H. M. Thomas, and G. E. Morfill, Phys. Rev. Lett. 99, 095002 (2007).

[5] M. Lampe, Glenn Joyce, G. Ganguli, and V. Gavrishchaka, Phys. Plasmas 7, 3851 (2000).

[6] R. Bingham and V. N. Tsytovich, IEEE Trans. Plasma Sci. 29, 158 (2001).

[7] R. Bingham and V. N. Tsytovich, Astron. Astrophys. 376, L43 (2001).

[8] Y. A. Mankelevich, M. A. Olevanov, and T. V. Rakhimova, Plasma Sources Sci. Technol. 17, 015013 (2008).

[9] Y.A. Mankelevich, M. A. Olevanov, A. F. Pal, T. V. Rakhimova, A. N. Ryabinkin, A.O. Serov, and A.V. Filippov, Phys. Plasmas Rep. 35, 191 (2009).

[10] C. H. Du, H. M. Thomas, A. V. Ivlev, U. Konopka, and G. E. Morfill, Phys. Plasmas 17, 113710 (2010).

[11] M. Rosenberg, J. Vac. Sci. Technol. A 14, 631 (1996).

[12] Robert L. Merlino, Phys. Plasmas 16, 124501 (2009).

[13] V. Nosenko, S. K. Zhdanov, S.-H. Kim, J. Heinrich, R. L. Merlino, and G.E. Morfill, Europhys. Lett. 88, 65001 (2009).

[14] S. Dap, D. Lacroix, R. Hugon, and J. Bougdira, J. Quant. Spectrosc. Radiat. Transfer (to be published).

[15] See Supplemental Material at http://link.aps.org/ supplemental/10.1103/PhysRevLett.109.245002 for the two movies performed during a typical experiment, they show: dust motion due to DDW (movie 1) and dust agglomeration (movie 2).

[16] S. Bardin, Ph.D. thesis, Université de Lorraine, 2012.

[17] F. Y. Huang, H. H. Hwang, and M. J. Kushner, J. Vac. Sci. Technol. A 14, 562 (1996).

[18] F. Y. Huang and M. J. Kushner, J. Appl. Phys. 81, 5960 (1997).

[19] C. K. Goertz, Rev. Geophys. 27, 271 (1989). 